\title{
The Use of Case-Based Reasoning for the Choice of Methods for Cleaning Exhaust Gases from Sulfur and Nitrogen Oxides
}

\author{
Yurii Beznosyk $^{1}$, Liudmyla Bugaieva ${ }^{2}$
}

1. The Department of Technical and Software automation, National Technical University of Ukraine "Igor Sikorsky Kyiv Polytechnic Institute”, UKRAINE, Kyiv, 37 Peremohy Ave, E-mail yu_beznosyk@ukr.net

2. The Department of Technical and Software automation, National Technical University of Ukraine "Igor Sikorsky Kyiv Polytechnic Institute”, UKRAINE, Kyiv, 37 Peremohy Ave, E-mail bugaeva_l@ukr.net

Abstract - At present, artificial intelligence methods are implemented in many computer programs. One such intelligent technique is Case-Based Reasoning (CBR). This approach is proposed by the authors for use in an intelligent system to choose cleaning methods for exhaust gases from nitrogen and sulfur oxides.

Keywords - case-based reasoning, case, exhaust gases, intelligent system, indexing, metric.

\section{Introduction}

Case-Based Reasoning (CBR) is an approach that allows to solve a new, unknown problem, using or adapting the solution of an already known problem, ie. using the experience already accumulated in solving such problems. The main concept in CBR is precedent (example, case) a structured representation of experience in the form of data and knowledge, which provides its further automated processing by specialized software systems. The method of inference on precedents or CBR is considered as one of the approaches of artificial intelligence or data mining.

\section{Case-Based Reasoning}

Let's see how CBR works. The life cycle of a CBR is shown in Fig. 1. Often CBR is called the $4 \mathrm{R}$ method [1-3]:

1. Retrieving - search for precedents;

2. Reusing - reuse;

3. Revising - revision (adaptation) of the selected use case;

4. Retaining - approval and preservation of the decision.

As you can see in fig. 1, the life cycle in CBR-based systems consists of these main stages or phases.

The critical phase in CBR is the first phase, namely the search for the most suitable old solutions for adaptation (Retrieving). The tricky part is that it's not always obvious how relevant the old case is. CBR considers similar problems to have similar solutions. The formalization of the similarity significantly affects the quality and speed of the CBR system. Note that the similarity score depends on the subject area. When using complex structures to represent use cases, assessing similarity can be very time-consuming. Also, an increase the base of cases leads to a decrease in the efficiency of extraction, since more cases have to be analyzed. To solve this problem, the socalled indexing of cases is used. Indexing problem is one of the main ones in CBR. It includes questions such as: the type of indexes to use and how to organize the index search. A trivial solution to the problem is to use all the input characteristics of a case as indices.

Various attempts are being made to use CBR in combination with various machine learning techniques. It can be seen that a number of works have recently appeared, where it is proposed to combine CBR with artificial neural networks of different types [4, 5]. For example, authors [5] propose an approach called NOD-CC, which is a combination of convolutional neural networks and case-based reasoning (CBR). 


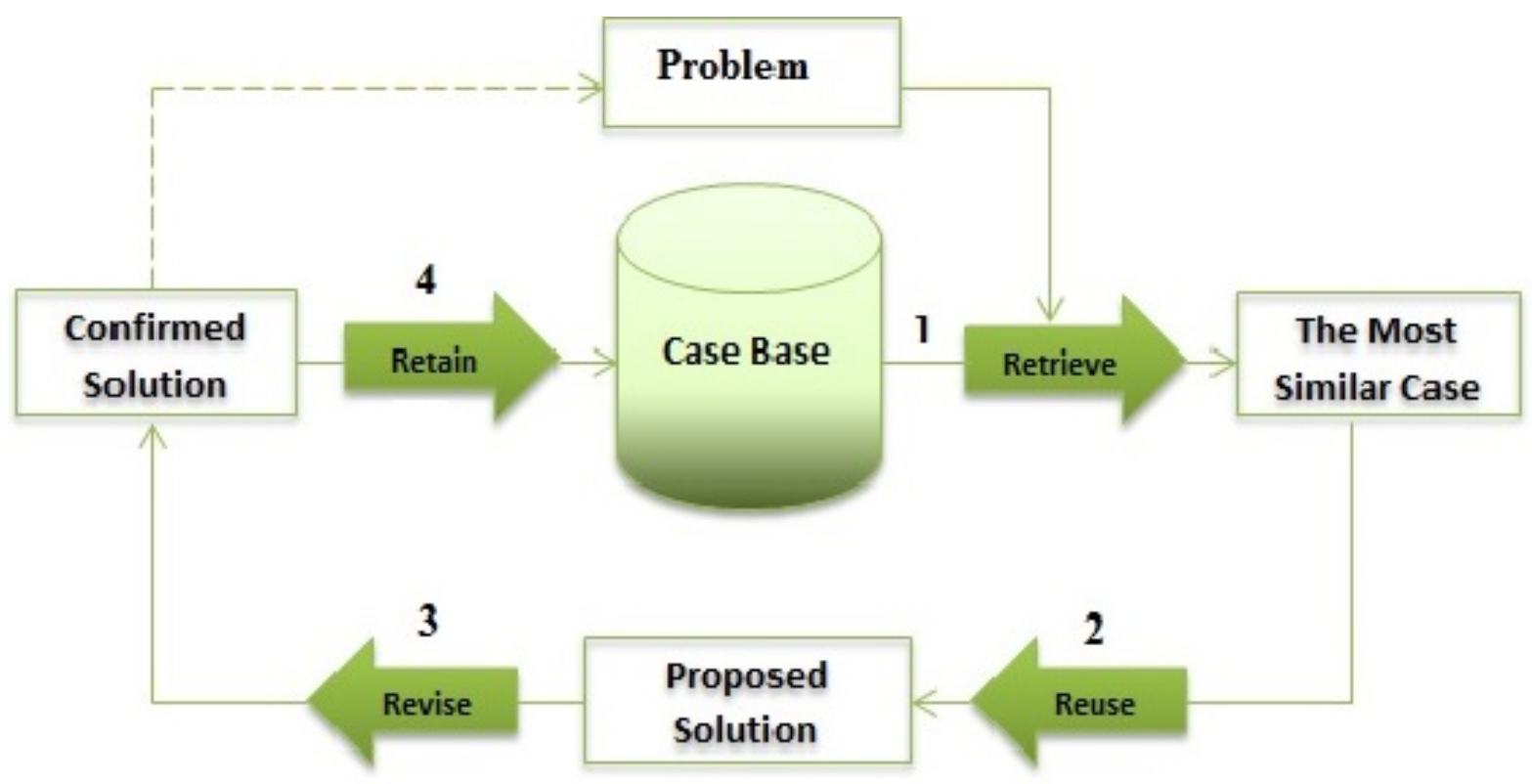

Fig.1. The life cycle of CBR.

A feature of the CBR approach and its implementation in software systems is its narrow specialization, that is, focus on solving specific problems in a limited subject area. As the analysis of the publication has shown, the applications based on the CBR approach for the tasks of chemical technology practically does not exist.

\section{Decision-making system for the choice of methods for cleaning exhaust gases from sulfur and nitrogen oxides}

This article shows the possibility of constructing an intelligent system using CBR to solve the urgent problem of chemical technology, namely, the choice of methods for cleaning exhaust gases from nitrogen and sulfur oxides. First of all, the collection of data for the formation of a base of precedents was carried out. The Case base keeps about 150 methods for cleaning exhaust gases from nitrogen and sulfur oxides that were formed by the specialists and the first version of the system was based on the rules [6]. However, the set of rules was not enough for searching relevant solution very often.

In the proposed system, it was possible to apply case characteristics as indices. The following characteristics of precedents were considered:

1. Degree of purification;

2. Initial concentration;

3. Temperature,

4. The presence of impurities;

5. Product;

6. Material consumption;

7. Energy consumption.

All these characteristics (attributes), except for the fourth and fifth, are given as numerical values with given units of measurement and can be easily normalized. The presence of impurities and the resulting product are categorical attributes with a certain set of values (classes).

The question now is, what metrics and method of similarity assessment will be appropriate to use in the developed system? The first two phases of the CBR lifecycle - Retrieving and Reusing - use the k-nearest neighbor method. There are many types of metrics that can be used 
to find close precedents - metrics like Heming, Mahalonobis, etc. The simpliest of them is Euclidean metric. It was used for estimating the distances between cases in the developed system.

During the third and fourth phases of CBR, the intervention of the decision maker is provided. The Revising phase - the adaptation of the relevant precedent is not necessarily automated procedure, rather it is a dialogue with an expert in a specific area. The process finished with the adoption of the found solution and the possible storage of this solution in the base of cases.

\section{Conclusion}

An intelligent decision-making system is proposed for the choice of methods for cleaning exhaust gases from sulfur and nitrogen oxides, based on the Case-Based Reasoning. The task was formalized: an indexing method and a metric for assessing similarity were selected. The work of the system was checked on test examples.

It is planned that the presented system will be a subsystem in the Decision Support System for the choice of methods for cleaning exhaust gases, which can use other decision-making methods [7]. The proposed CBR-based methodology, which has proven its effectiveness in making decisions on the choice of cleaning methods, can be extended to other technological problems, where enough solution options have been accumulated in the past. Of course, adapting old solutions is unlikely to lead to innovation, but finding solutions based on proven experience will always correspond to less uncertainty.

\section{References}

[1] Kolodner J. (1993) Case-Based Reasoning. San Mateo: Morgan Kaufmann. 668 p.

[2] Kolodner J. (2005) Retrieving events from case memory: A parallel implementation. // In: Proceedings from the Case-based Reasoning Workshop, DARPA, Clearwater Beach. P. 233-249.

[3] Aamodt A. (1994) Explanation-driven case-based reasoning. // In: Wess, S., Althoff, K.-D., Richter, M.M. (eds.) European Workshop on Case-Based Reasoning. Lecture Notes in Computer Science (Lecture Notes in Artificial Intelligence). Vol. 837. P. 274-288. https://doi.org/10.1007/3-540-58330-0_93.

[4] Turner J. T., Floyd M. W., Gupta K. \& Oates T. (2019) NOD-CC: A Hybrid CBR-CNN Architecture for Novel Object Discovery. // In: Bach K., Marling C. (eds) Case-Based Reasoning Research and Development. Lecture Notes in Computer Science. Springer, Cham. ICCBR 2019. Vol 11680. https://doi.org/10.1007/978-3-030-29249-2_25.

[5] Amin K., Kapetanakis S., Althoff K.-D., Dengel A. \& Petridis M. (2018) Answering with cases: a CBR approach to deep learning. // In: Cox, M.T., Funk, P., Begum, S. (eds.) Springer, Cham. ICCBR 2018. LNCS (LNAI), Vol. 11156. P. 15-27. https://doi.org/10.1007/978-3-030-01081-2_2.

[6] Bugaeva L. N., Beznosik Yu. A., Statjukha G. A. \& Kvitka A. A. (1996) An application of expert system to choice, simulation and development of gases purification processes. Journal Computers \& Chemical Engineering, Vol. 20, Suppl., P. S402-S406. https://doi.org/10.1016/0098-1354(96)00077-4.

[7] Bugaeva L. N., Bojko T. V. \& Beznosyk Yu. A. (2017) System analysis of chemicaltechnological systems, Kyiv: Interservice. 253 p. 